\title{
InGaN stress compensation layers in InGaN/GaN blue LEDs with step graded electron injectors
}

\author{
V. Sheremet ${ }^{\text {a, }{ }^{*}, \text { N. Gheshlaghi }}{ }^{\text {a }}$, M. Sözen ${ }^{a}$, M. Elçi ${ }^{\text {a, b }}$, N. Sheremet ${ }^{\text {a, c }}$, \\ A. Aydınlı a, d, *, I. Altuntaş ${ }^{\text {e, K. Ding }}{ }^{\mathrm{f}}$, V. Avrutin ${ }^{\mathrm{f}}$, Ü. Özgür ${ }^{\mathrm{f}}$, H. Morkoç ${ }^{\mathrm{f}}$ \\ a Advanced Research Laboratories, Department of Physics, Bilkent University, Ankara, 06800 Turkey \\ ${ }^{\mathrm{b}}$ Institute of Applied Mathematics, Middle East Technical University, Ankara, 06800 Turkey \\ ${ }^{\mathrm{c}}$ Institute of Physics, NAS of Ukraine, Kyiv, 03680 Ukraine \\ d Department of Electrical and Electronics Engineering, Uludağ University, Bursa, 16059 Turkey \\ e Department of Nanotechnology Engineering, Cumhuriyet University, Sivas, 58140 Turkey \\ ${ }^{\mathrm{f}}$ Department of Electrical and Computer Engineering, School of Engineering, Virginia Commonwealth University, Richmond, VA, 23284- \\ 3068, USA
}

\section{A R T I C L E I N F O}

\section{Article history:}

Available online 3 February 2018

\section{Keywords:}

Light-emitting diode

Step-graded electron injector

Stress compensation layer

InGaN/GaN

\begin{abstract}
A B S T R A C T
We investigate the effect of InGaN stress compensation layer on the properties of light emitting diodes based on InGaN/GaN multiple quantum well (MQW) structures with stepgraded electron injectors. Insertion of an InGaN stress compensation layer between n-GaN and the step graded electron injector provides, among others, strain reduction in the MQW region and as a result improves epitaxial quality that can be observed by 15 -fold decrease of V-pit density. We observed more uniform distribution of In between quantum wells in MQW region from results of electro- and photoluminescence measurement. These structural improvements lead to increasing of radiant intensity by a factor of $1.7-2.0$ and enhancement of LED efficiency by $40 \%$.
\end{abstract}

() 2018 Elsevier Ltd. All rights reserved.

\section{Introduction}

Gallium nitride is a wide band gap semiconductor that is widely used for ultraviolet, blue, and green light emitting diodes (LEDs) intended for backlighting, automotive headlights, communications, wireless optical networking (Li-Fi) and general lighting. Standard InGaN/GaN LED dies consist of InGaN multiple quantum well (MQW) active regions separated by GaN or low In content InGaN barriers sandwiched between highly doped n- and p-type gallium nitride layers to confine the carriers [1]. Despite extensive use of InGaN/GaN MQWs in blue LEDs, there still remain physical and technical problems which need to be overcome. A central limiting factor is the lack of inexpensive large-area bulk GaN substrates for epitaxial growth [2]. Therefore, vast majority of InGaN LEDs are grown on foreign substrates with associated structural defects and residual strain. In view of high temperatures and chemically aggressive environment required for the growth of GaN epitaxial layers, high thermal and chemical stability of sapphire together with its low cost and availability make it a natural substrate choice for GaN-based semiconductor devices. However, large lattice mismatch between sapphire and GaN ( 16\%) [3] results in high stress that partially relaxes through defect generation, giving rise to high densities of threading dislocations (TDs). GaN based

\footnotetext{
* Corresponding authors. Advanced Research Laboratories, Department of Physics, Bilkent University, Ankara, 06800 Turkey.

E-mail addresses: sheremet@bilkent.edu.tr (V. Sheremet), atillaaydinli@uludag.edu.tr (A. Aydınlı).
} 
device epitaxy requires extra effort to reduce lattice mismatch induced defect densities that can cause leakage currents, siphoning carriers from radiative processes. The TD density can be lowered to $\sim 10^{8} \mathrm{~cm}^{-2}$ and below only with the use of special techniques such as epitaxial lateral overgrowth with in situ deposited $\mathrm{Si}_{3} \mathrm{~N}_{4}$ nanoporous mask (frequently dubbed as nano-ELO technique) [4-6].

The reasons for efficient recombination in InGaN/GaN QWs despite the presence of high defect densities that would lead to rapid degradation of LED characteristics in other III-V semiconductors such as GaAs and InP are still being debated. The most commonly proposed reason is localization of charge carriers in the QW planes, not allowing them to take part in nonradiative recombination [7-9]. There is some evidence that high lattice mismatch between the InN and GaN binaries (11\%) leads to strain in the LED active regions and hence the amount of indium that can be incorporated into InGaN layers is somewhat limited [10-12]. Partial strain relaxation in InGaN QWs can lead to In-rich nanocluster formation through local compositional fluctuations in InGaN, resulting in strong carrier localization in the QW plane [7-9,11,13-15]. This prevents capture of charge carriers by nonradiative recombination centers associated with high density of threading dislocations [11,13-15]. Although, high degree of localization can hurt the LED efficiency by reducing active LED area. On the other hand, 3D atom probe measurements [16-18] suggest that InGaN in QWs is a random alloy, and hence, In rich regions do not exist. An alternative explanation of the observed high efficiency in the presence of large number of defects involves the antilocalization theory $[19,20]$. Due to smaller QW widths, and therefore, higher confinement in the V-shaped hexagonal pits that appear on the threading dislocations, a potential barrier of the order of $400 \mathrm{meV}$ is formed in the vicinity of the pits. This barrier keeps charge carriers away from the dislocations and the associated defects and mitigates nonradiative recombination [19,20].

Growth of InGaN/GaN LED structures on c-plane sapphire surfaces, as compared to semi- and non-polar orientations, has the advantage of achieving lower defect densities. However, a major disadvantage of this polar orientation is the presence of high polarization fields [11,21]. Large spontaneous and piezoelectric fields in the biaxially compressive strained InGaN QWs lead to separation of electrons and holes within a given QW [10,11,21,22]. Reduced carrier wavefunction overlap caused by the Quantum Confined Stark Effect (QCSE) decreases the recombination rate and emission efficiency, particularly, at low injection [23-25]. To mitigate this problem, the thickness of QWs in InGaN/GaN polarized heterostructures is limited to 2-3 nm [24]. QCSE can also be mitigated by screening with the help of doping quantum well barriers, using nonpolar or semipolar orientations, and replacing the GaN quantum barriers with InGaN $[21,25,26]$.

Another alternative approach to minimize the effect of QCSE while also enhancing InN solubility in InGaN/GaN QWs is employing an additional low In content InGaN layer inserted between n-GaN and the MQW region of the LED structure, frequently dubbed as pre-strained growth [12,21,27-34]. Growth of this thick low indium content layer below the MQW active region results in reduction of strain in the QWs and improvement in the active region quality. As a stress compensation layer (SCL) superlattices based on InGaN have also been used [23,25,35]. In overall, SCL can pave the way to more uniform inplane indium composition in the QWs [36], reduction of threading dislocations which have been quantified in terms of V-pit densities [23,25,35,37], improving electrostatic discharge properties [36] and improving current spreading by acting as an electron reservoir [34,36,38]. As a result, LEDs featuring a SCL exhibit much shorter carrier lifetimes [36,39] and show enhanced internal quantum efficiencies [25] as well as increased optical power output [34,39]. It should be noted that most of the works mentioned above are devoted to research conducted on small die sizes with dimensions of the order of $300 \times 300 \mu \mathrm{m}^{2}$. However, understanding the nature of the above mentioned effects is of great importance for high power LEDs that in general have dimensions on the order of $1 \times 1 \mathrm{~mm}^{2}$.

A further problem that limits the performance of InGaN/GaN LEDs is the ballistic and quasi-ballistic electron overflow through the MQW active region [40,41]. Commonly, this problem is overcome using an electron blocking layer of a wider band gap material such as AlGaN between MQW region and p-GaN [1]. However, due to the accompanying valence band discontinuity that can exceed $0.08 \mathrm{eV}$ for the commonly used $\mathrm{Al}_{0.15} \mathrm{Ga}_{0.85} \mathrm{~N}$ layer, an electron blocking layer can also compromise hole injection to the active region [42]. Use of an InGaN staircase, step-graded, or continuously graded electron injector, as proposed in Refs. [40-43], is expected to cool electrons before their injection to the active region, preventing their overflow without impeding the hole transport. The electron injector layer, because of its gradually increasing indium content, can compensate stress in quantum well region as well.

In this work, we report on the effects of InGaN SCL on the electrical and optical performance of InGaN blue LEDs with stepgraded electron injectors (SGEI) ( $\operatorname{In}_{\mathrm{x}} \mathrm{Ga}_{1-\mathrm{x}} \mathrm{N}$ with $\mathrm{x}$ varying from 0.04 to 0.1 in 7 steps). We studied the effect of SCL on properties of epitaxial structures as well as properties of LED dies of $1 \times 1 \mathrm{~mm}^{2}$ area. We demonstrate that using SCL in LED epiwafers can decrease strain and improve indium incorporation in QWs, and as a result, reduce defect density in the active layers, and therefore, enhance the radiative recombination efficiency of blue InGaN LEDs.

\section{Experimental}

InGaN MQW based LED structures used in this work were grown by metalorganic chemical vapor deposition (MOCVD) technique on c-plane sapphire substrates. To improve crystal quality and decrease threading dislocation density, epitaxial lateral overgrowth with the aid of in-situ $\mathrm{Si}_{3} \mathrm{~N}_{4}$ masks was utilized [4-6]. Epitaxial structure consists of 10- $\mu \mathrm{m}$-thick undoped GaN, followed by $4-\mu \mathrm{m} n-G a N\left(3 \cdot 10^{18} \mathrm{~cm}^{-3}\right)$. For LED A, a 7 step SGEI $\operatorname{In}_{\mathrm{x}} \mathrm{Ga}_{1-\mathrm{x}} \mathrm{N}(\mathrm{x}=0.04$ to $0.1 \mathrm{with} 3 \mathrm{~nm}$ thickness for each step) was grown directly on the n-GaN layer. For LED B, a $60 \mathrm{~nm} \operatorname{In}_{0.03} \mathrm{Ga}_{0.97} \mathrm{~N}$ SCL was inserted between n-GaN and SGEI. For both samples, MQW region consists of $6 \times 2 \mathrm{~nm} \mathrm{In}{ }_{0.15} \mathrm{Ga}_{0.85} \mathrm{~N}$ QWs separated by $3 \mathrm{~nm}$-thick $\operatorname{In}_{0.06} \mathrm{Ga}_{0.94} \mathrm{~N}$ barriers. A $100 \mathrm{~nm}$ thick p-GaN $\left(4 \cdot 10^{17} \mathrm{~cm}^{-3}\right)$ completes the device structure. Square mesas with interdigitated electrode patterns were 
fabricated with different contact pad to p-n junction edge distances ( $d=50,100,150$ and $200 \mu \mathrm{m}$ ). They were formed by inductively coupled plasma (ICP) etching using $\mathrm{BCl}_{3}: \mathrm{Cl}_{2}$ chemistry. LED dies edges were passivated by $250 \mathrm{~nm}$ plasmaenhanced chemical vapor deposition (PECVD) grown $\mathrm{SiO}_{2}$ immediately after ICP etching. Ohmic contacts to n-GaN were formed by using $\mathrm{Ti}(30 \mathrm{~nm}) / \mathrm{Al}(50 \mathrm{~nm}) / \mathrm{TiB}_{2}(50 \mathrm{~nm}) / \mathrm{Au}(70 \mathrm{~nm})$ stack subjected to rapid thermal annealing (RTA) in $\mathrm{N}_{2}$ at $\mathrm{T}=800^{\circ} \mathrm{C}$ for $30 \mathrm{~s}$. For current spreading layer and ohmic contact to p-GaN, Ni(5 nm)/Au(5 nm)/ITO(120 nm) stacks were used with $\mathrm{Ti}(30 \mathrm{~nm}) / \mathrm{Au}(50 \mathrm{~nm})$ contact pads. The structures of fabricated LEDs are shown in Fig. 1.

Electrical properties of fabricated LED dies were measured using a Keithley 2430 source and measurement unit. Electroluminescence (EL) spectra and power output of LEDs were measured by Instrument Systems spectrometer CAS 140CT. Photoluminescence (PL) measurements of LED wafers were performed by using a He-Cd laser with $325 \mathrm{~nm}$ emission wavelength and maximum power of $50 \mathrm{~mW}$. The PL spectra were collected using Horiba Jobin Yvon U1000 double monochromator and a CCD detector.

\section{Results and discussion}

Spatial distributions of optical emission of biased LED dies of type A and B recorded under the same light collection conditions using an optical microscope are presented in Fig. 2. Light output intensity from LED B is significantly higher than that from LED A. While the light emission between the n-type contacts and p-type fingers is homogenous at the macroscopic scale, there are differences at the microscopic scale. Sample A without SCL shows peculiar dark lines mostly perpendicular to the contact fingers while the sample with the InGaN SCL (LED B) does not demonstrate such features. Dark lines can be associated with nonradiative recombination centers and can be construed as evidence of dislocations or In compositional fluctuation due to compressive strain in the InGaN quantum wells for samples without SCL [11,13-15].

In Fig. 3a, representative I-V characteristics of dies with $\mathrm{d}=150 \mu \mathrm{m}$ are shown. Average series resistance values for these LEDs were $11 \Omega$ and $13 \Omega$ and turn on voltage was equal to 2.8 and $3.3 \mathrm{~V}$ for LEDs $\mathrm{A}$ and $\mathrm{B}$, respectively. I-V characteristics from dies with different $d$ values exhibit slight variations, and the series resistances of LED dies A and B vary within $\pm 18 \%$ with changing $d$. In spite of the small differences in electrical properties of LEDs A and $B$, there is a notable difference in radiant intensities of these devices (Fig. 3b). Radiant intensity from LED B with SCL is 1.7-2.0 times higher than that for LED A without SCL (Fig. 3b). Consequently, the peak efficiency of LEDs with SCL is nearly $40 \%$ higher (Fig. 3b). The efficiency roll-off at 33 A/ $\mathrm{cm}^{2}$ current density was calculated as $22 \%$ for both LEDs A and B, indicating that the efficiency droop does not depend on the existence of SCL for samples investigated in this study. Electrical and radiant LED dies properties measurement results indicate that nonradiative processes have significant role for LED without SCL.

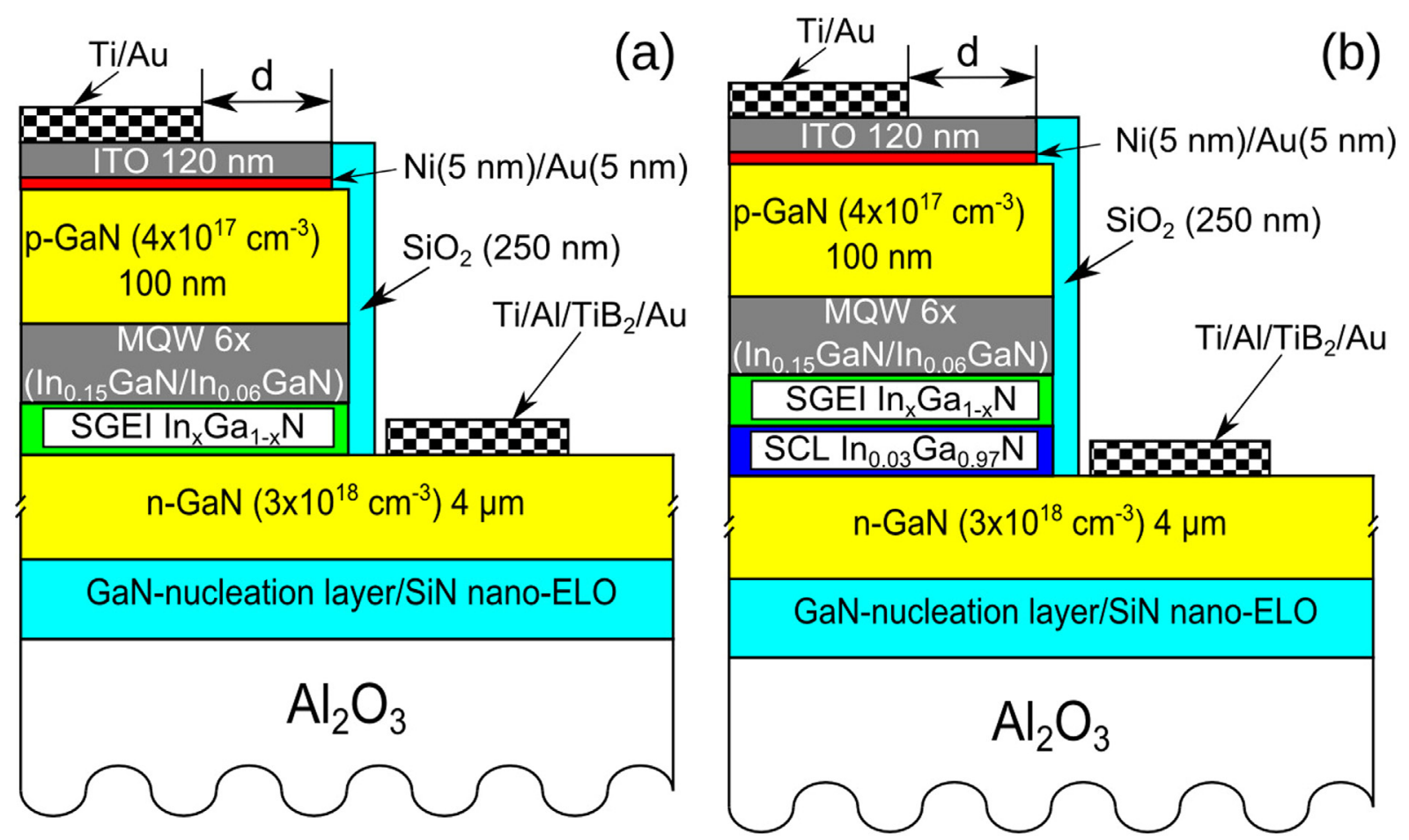

Fig. 1. Structures of LED dies without (a) and with (b) $\operatorname{In}_{0.03} \mathrm{Ga}_{0.97} \mathrm{~N}$ stress compensation layer. The distance from the contact pad to the edge of the mesa is labeled, d. 

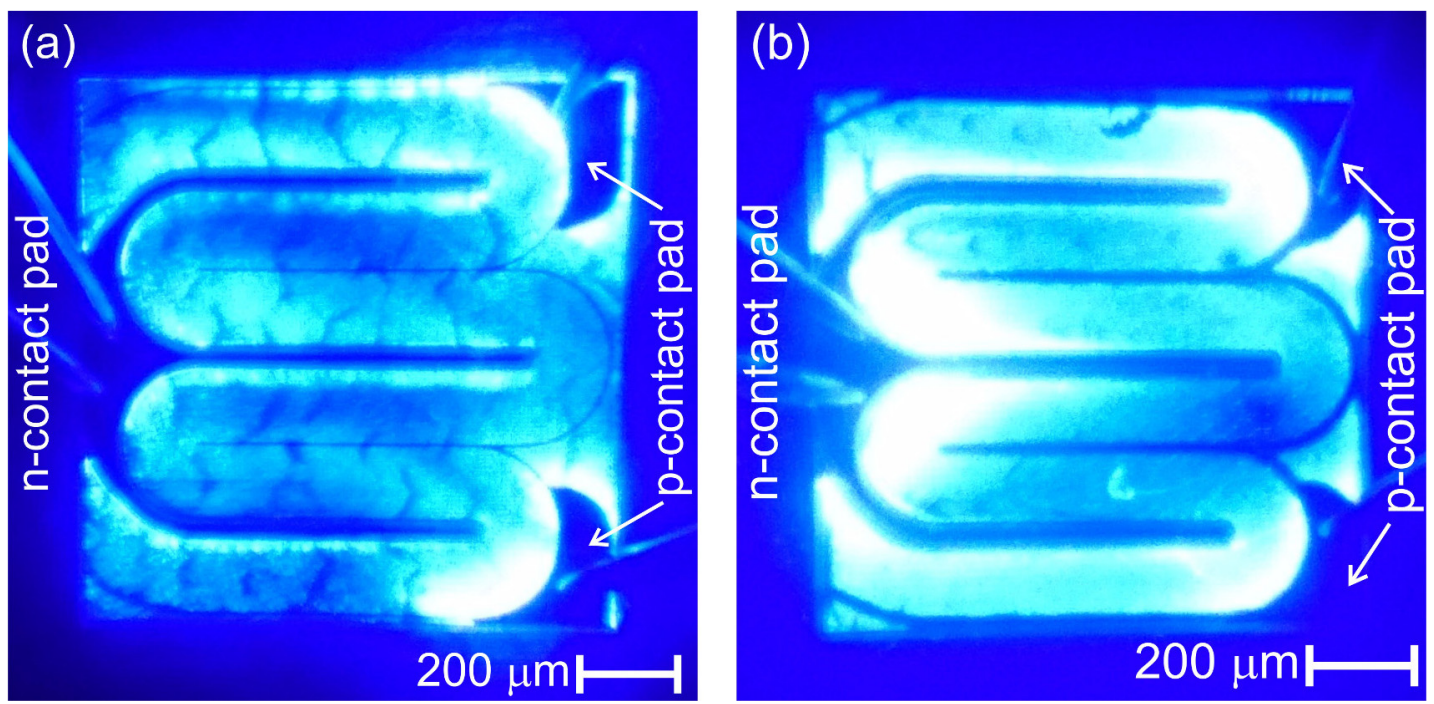

Fig. 2. Optical microscope images of emission from LED dies without stress compensation layer (LED A) (a) and with stress compensation layer (LED B) (b).

The differences discussed above in light emission properties of LEDs can be attributed to higher stress in samples without SCL that can result in increased QCSE and defect density or higher In fluctuation in the QW InGaN compounds causing larger charge carrier localization. Carrier localization [7-9,11,13-15] in the plane of the QWs as a result of In fluctuation in InGaN compounds can decrease active LED die area and QCSE may be responsible for decreasing of charge carrier recombination that in turn causes a decrease in efficiency [13,15,23-25]. To reveal possible mechanisms responsible for this behavior, we carefully analyzed electroluminescence spectra of LED structures at different injection levels.

As one can see from Fig. 4 insertion of SCL leads to a slight blue-shift in EL peaks, which may be the result of lower In content in QW of LED B although conditions for epiwafer growth were the same for both LEDs. EL spectra of LED A at low currents (Fig. 4b) display two distinct peaks at 447 and $470 \mathrm{~nm}$, with the latter being dominant at very low injection $(<18 \mathrm{~mA})$. With increasing current, the low energy peak slightly blue-shifts and can be observed as a clear shoulder on the EL spectrum up to $150 \mathrm{~mA}$. At low currents, emission mostly originates from the top QWs due to low mobility of holes. This suggests that QWs close to p-GaN have higher In incorporation. Stress relaxation by defect generation can be responsible for drastic increasing of In incorporation in QWs [44]. At currents $>40 \mathrm{~mA}$, the high energy peak becomes dominant and slightly blueshifts with increasing current up to $146 \mathrm{~mA}$. With increasing injection, deeper QWs start contributing to emission. In the case of LED B, only a single peak is apparent at $442 \mathrm{~nm}$ even at low currents.

Fig. 5 displays peak wavelength for LEDs A and $B$ as a function of injection current. With increasing injection current from 0 to $150 \mathrm{~mA}$, the peak wavelength blue-shifts for both LEDs. For LED A the peak wavelength is shifted from 446.8 to $443.5 \mathrm{~nm}$ (by $21 \mathrm{meV}$ ), and LED B that has SCL exhibits a blue-shift from $442.0 \mathrm{~nm}$ to $440.3 \mathrm{~nm}$ (by $11 \mathrm{meV}$ ). This blue-shift can be a result of QCSE screening as well as band filling $[10,28,45]$. We emphasize that blue-shift with increasing injection in LED A is twice that in LED B, which suggests smaller strain and QCSE in LED B [45]. With increasing current injection beyond $150 \mathrm{~mA}$, EL peaks red-shift from $443.5 \mathrm{~nm}$ to $444.5 \mathrm{~nm}$ (by $6.3 \mathrm{meV}$ ) and from $440.3 \mathrm{~nm}$ to $441.6 \mathrm{~nm}$ (by $8.3 \mathrm{meV}$ ) for LEDs A and B, respectively. This red-shift can be attributed to Joule heating of dies, which increases the dispersion of the carrier distribution, and to bandgap renormalization caused by high carrier density in the active region $[10,28,45]$.

To understand the observed difference in emission wavelength from LEDs with and without SCL and the role of the stress in decreasing the efficiency of LEDs with SGEI and without SCL, we performed photoluminescence (PL) experiments under $325 \mathrm{~nm}$ excitation from a He-Cd laser. PL spectra at $7 \mathrm{~K}$ and $300 \mathrm{~K}$ are presented in Fig. 6 . The emission below $380 \mathrm{~nm}$ (not shown) is associated with emission from SCL (LED B) as well as the n-GaN template. The use of the high-pass filter to block the scattered laser light does not allow to distinguish the corresponding peaks accurately. PL features observed near $405 \mathrm{~nm}$ $(3.07 \mathrm{eV})$ for the sample without SCL and $410 \mathrm{~nm}(3.03 \mathrm{eV})$ for the sample with SCL are associated with SGEI [23]. These observations indicate higher In incorporation in SGEI of LED B with SCL that can be a result of lower strain in the SGEI.

QW emission at $7 \mathrm{~K}$ give peaks at $435 \mathrm{~nm}(2.858 \mathrm{eV})$ and $444 \mathrm{~nm}(2.800 \mathrm{eV})$ for LEDs A and B, respectively. The difference of $58 \mathrm{meV}$ can be attributed to a higher In content in QWs of LED B, by more than $1 \%$ according to [46]. Full width at half maximum (FWHM) values of PL peaks from QWs are $15.3 \mathrm{~nm}$ and $12.6 \mathrm{~nm}$ (i.e., 80 and $100 \mathrm{meV}$ ) for LEDs A and B, respectively. The narrower peak and 44\% higher PL intensity for LED B can be attributed to better crystal quality and homogeneity in In distribution.

As the temperature is increased to $300 \mathrm{~K}$, we observe a negligible red-shift of the PL peak for quantum wells to $445 \mathrm{~nm}$ and a 34 times drop in PL intensity, compared to that at $7 \mathrm{~K}$, for LED B. With increasing temperature, the QW PL peak in LED A redshifts more $(5 \mathrm{~nm})$ and reduces more drastically (57 times) compared to LED B. Larger PL red-shift confirms higher strain in 

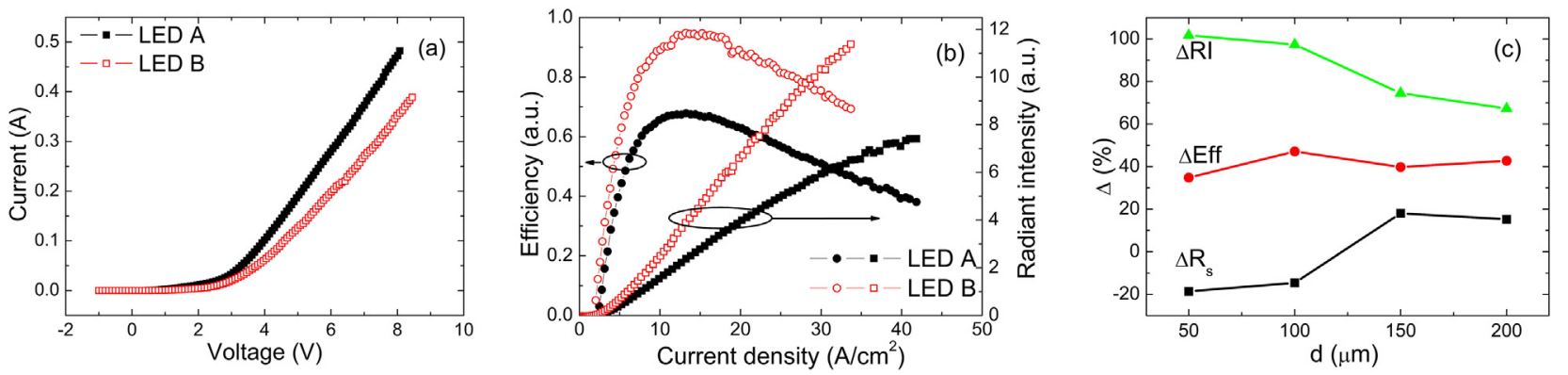

Fig. 3. Representative I-V characteristics (a), efficiency and radiant intensity dependencies on current density of LEDs (b) without (solid symbols) and with (hollow symbols) SCL for $\mathrm{d}=150 \mu \mathrm{m}$ electrode separation distance and (c) percentile changes compared to LED A in series resistances $\left(\Delta R_{s}\right)$, peak efficiencies ( $\Delta$ Eff) and radiant intensities $(\Delta R I)$ of LED B dies with $d=50,100,150$ and $200 \mu \mathrm{m}$. 

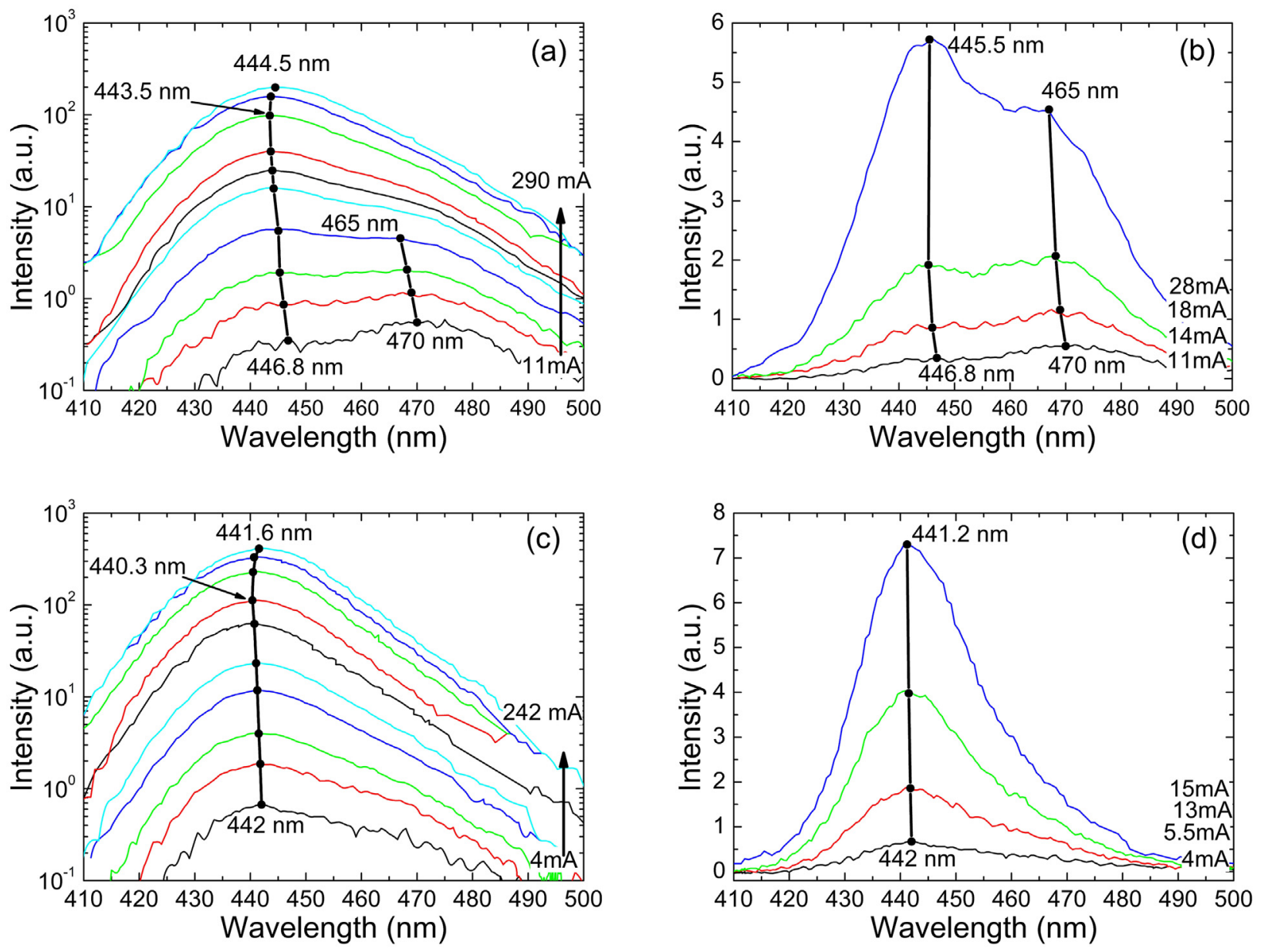

Fig. 4. EL spectra of LEDs without (LED A) (a, b) and with (LED B) (c,d) stress compensation layer measured at currents 4-290 mA. The right panels show the EL spectra at low currents in linear scale.

LED A epiwafer. A shoulder peak around $460-470 \mathrm{~nm}$, which can be attributed to phase separation in MQWs [47] was observed for both LEDs in the 7K PL spectra. A broad yellow band emission, attributed to recombination at extended crystal defects; dislocations, grain boundaries, gallium and nitrogen vacancies and related complexes in GaN [37,48], is also visible for both LEDs.

Screening of polarization field (e.g., reducing QCSE) via carrier injection is expected to result in increased PL peak intensity accompanied by a blue-shift [37]. In our case, insertion of SCL results in increasing of PL peak intensity with a red-shift. This behavior is opposite of what is expected for the elimination of QCSE. Therefore, red-shift of the PL peak suggests higher In content in the QWs of LED B. Obtained PL spectra peaks positions are in contrast with the EL spectra. EL spectra peaks show shorter emission wavelength for the sample with SCL (LED B). The observed discrepancy can be explained by the variation in In composition across the QWs and the fact that the EL spectrum originates mainly from the QWs closer to p-GaN because of short migration distance of holes, especially at lower currents, and the PL spectrum has equal contributions from the QWs that are further from the episurface [12,27,32].

Defect generation is one of the avenues for strain relaxation. From a study of the epitaxial surface features, one can see that the V-pit density in LED A $\left(9 \cdot 10^{5} \mathrm{~cm}^{-2}\right)$ is much larger than that in LED B $\left(6 \cdot 10^{4} \mathrm{~cm}^{-2}\right)$ (Fig. 7). Higher rms value of the surface roughness obtained from atomic force microscopy (AFM) measurements of $2.5 \times 2.5 \mu \mathrm{m}$ area (not shown) for LED A (1.36 nm) compared to that for LED B $(0.8 \mathrm{~nm})$ is one more evidence of higher defect density in LED without SCL.

The results presented above, in aggregate, suggest that the $60 \mathrm{~nm} \operatorname{In}_{0.03} \mathrm{Ga}_{0.97} \mathrm{~N}$ SCL allows to relax stress by defect generation after exceeding the critical thickness. According to literature [49,50] critical thickness for alloy with $3 \%$ of In is lower than $60 \mathrm{~nm}$. As a result of stress relaxation in SCL, stress in the SGEI is lower and allows to incorporate 10\% of In in LED B instead of $9 \%$ as in the case of LED A. As a result of stress relaxation, distribution of In in different QWs of LED B MQW active region is more uniform as we can see from PL results. Stress in MQW area increases with increasing number of QWs, resulting in lower In incorporation in top QWs as can be seen from blue-shift of EL spectra peaks. In the case of LED A without SCL, 


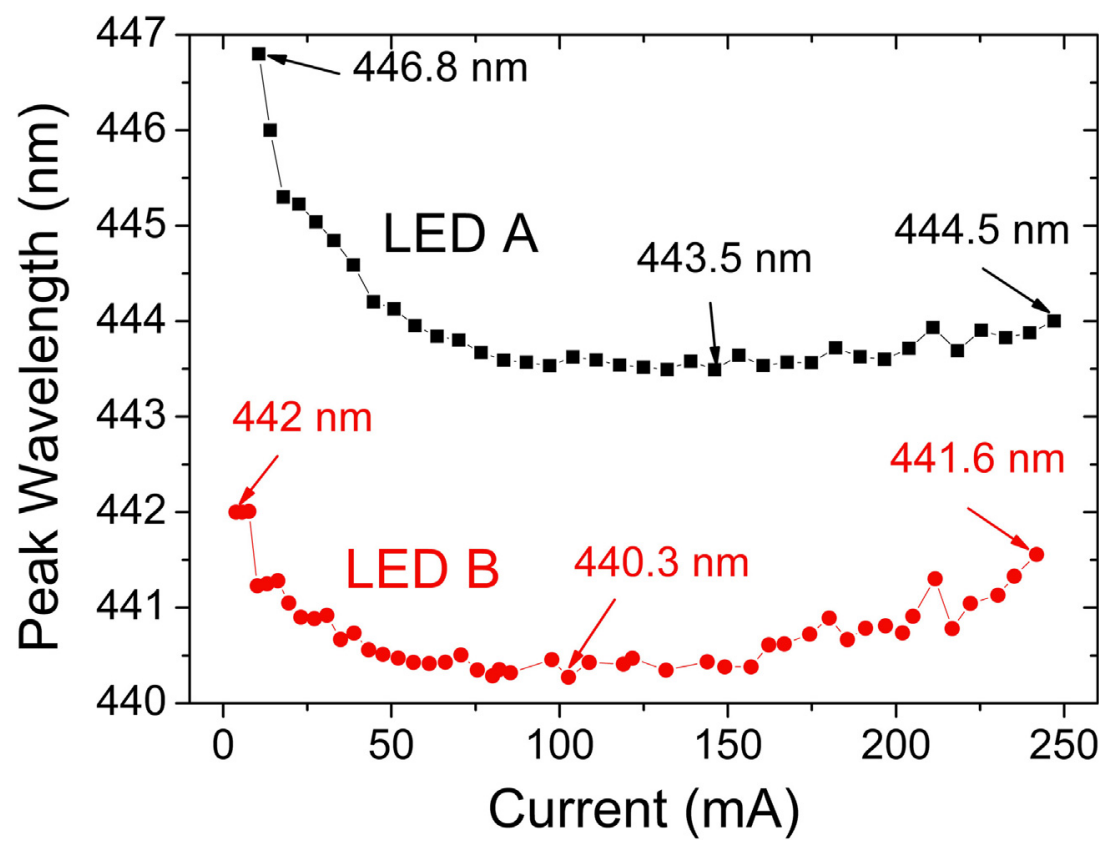

Fig. 5. Peak wavelength of LEDs with (LED B) and without (LED A) stress compensation layer.

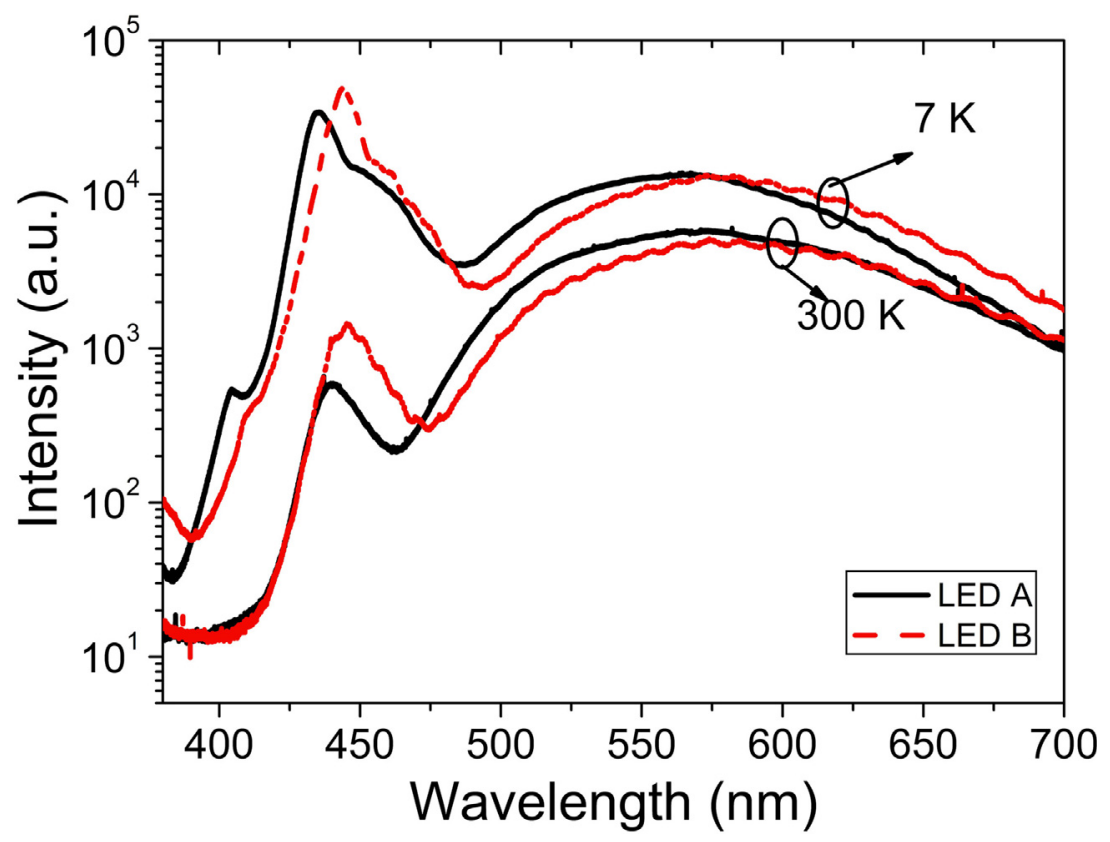

Fig. 6. Photoluminescence spectra of LED samples with (LED B) and without (LED A) SCL under $325 \mathrm{~nm}$ excitation from a He-Cd laser.

relaxation takes place after exceeding the critical thickness in MQW region. This results in higher In incorporation in relaxed top QWs. EL spectra at low currents exhibit a $470 \mathrm{~nm}$ dominant peak, which originates from the top QWs due to limited mobility of holes in GaN based LEDs and supports the expectation of high In incorporation (up to 20\%) in top QWs. 15 times higher V-pit density on the surface of LED A suggests that this relaxation is associated with defect generation in the MQW region.

In aggregate one can see that using of SCL for LED structures with SGEI decreases stress preventing relaxation by defect generation in MQW region, enhancing LED optical output and its efficiency. 

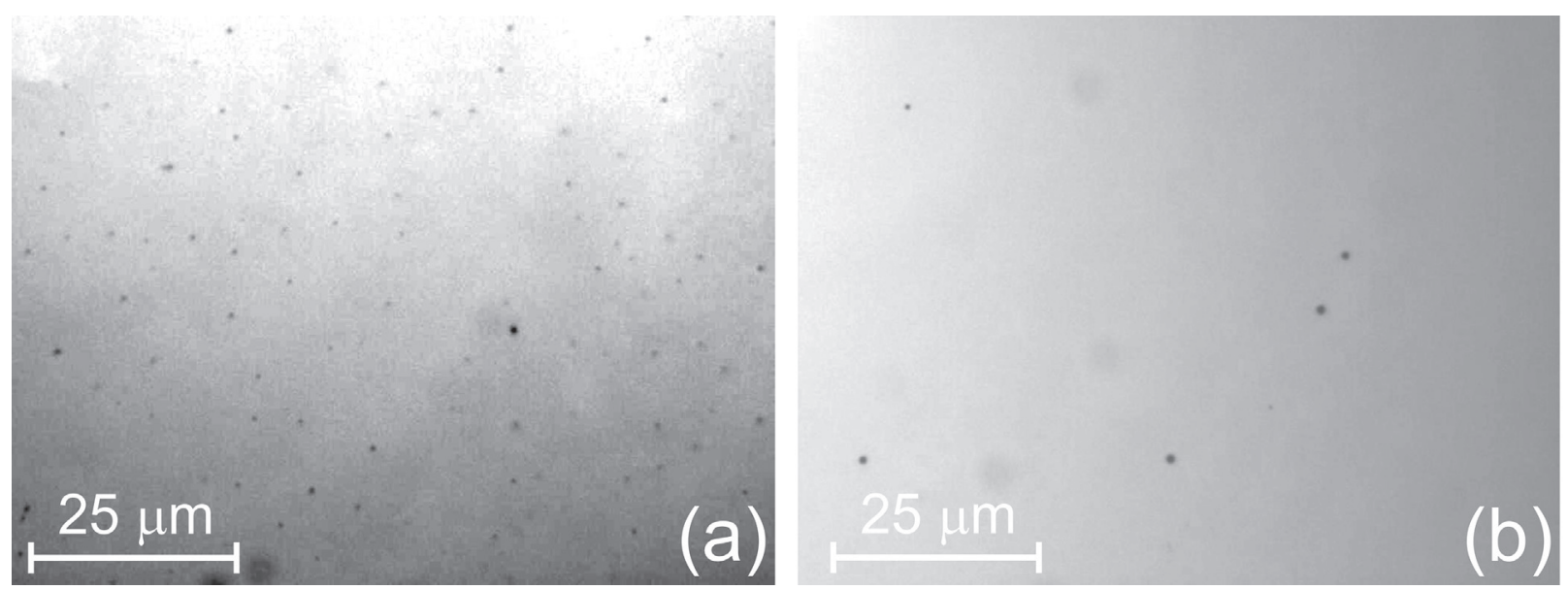

Fig. 7. Optical microscope images of wafer surfaces of (a) LED A and (b) LED B.

\section{Conclusions}

We have investigated InGaN/GaN MQW blue LED structures with step graded electron injectors used for electron cooling and thus mitigation of electron overflow. Specifically, we studied the effect of a $60 \mathrm{~nm}$ thick $\operatorname{In}_{0.03} \mathrm{Ga}_{0.97} \mathrm{~N}$ stress compensation layer grown between n-GaN and the SGEI region, on the properties of LEDs. Efficiency was found to be $40 \%$ higher for LEDs with SCL due to improved crystallinity. EL and PL measurements revealed that SCL improves In incorporation, decreases In clustering, and decreases V-pit defect density by a factor of 15.

\section{Acknowledgements}

Financial support for this work is provided by the Scientific and Technological Research Council of Turkey (Grant No: 113G042). Ismail Altuntaş acknowledges the Ph.D. Grant support from TUBITAK. We thank UNAM-National Nanotechnology Research Center at Bilkent University for access to fabrication and characterization equipment.

\section{References}

[1] E.F. Schubert, Light-emitting Diodes, 2-nd ed., Cambridge University Press, Cambridge, 2006.

[2] V. Avrutin, D.J. Silversmith, Y. Mori, F. Kawamura, Y. Kitaoka, H. Morkoç, Bulk GaN and AlN: progress and challenges, Special Issue of Proc. of IEEE on "Challenges and Opportunities in GaN and ZnO Devices and Materials" 98 (2010) 1302-1315.

[3] H. Amano, M. Iwaya, T. Kashima, M. Katsuragawa, I. Akasaki, J. Han, S. Hearne, J.A. Floro, E. Chason, J. Figiel, Stress and defect control in GaN using low temperature interlayers, Jpn. J. Appl. Phys. 37 (1998) L1540-L1542.

[4] H. Morkoç, Handbook of Nitride Semiconductors and Devices, Vol. 1: Materials Properties, Physics and Growth, WILEY-VCH Verlag GmbH \& Co. KGaA, Weinheim, 2008.

[5] J. Xie, Ü. Özgür, Y. Fu, X. Ni, H. Morkoç, C.K. Inoki, T.S. Kuan, J.V. Foreman, H.O. Everitt, Low dislocation densities and long carrier lifetimes in GaN thin films grown on a $\mathrm{SiN}_{\mathrm{x}}$ nanonetwork, Appl. Phys. Lett. 90 (2007), 041107.

[6] M.J. Kappers, R. Datta, R.A. Oliver, F.D.G. Rayment, M.E. Vickers, C.J. Humphreys, Threading dislocation reduction in (0001) GaN thin films using SiN $\mathrm{x}_{\mathrm{K}}$ interlayers, J. Cryst. Growth 300 (2007) 70-74.

[7] M. Filoche, M. Piccardo, Y.-R. Wu, C.-K. Li, C. Weisbuch, S. Mayboroda, Localization landscape theory of disorder in semiconductors. I. Theory and modeling, Phys. Rev. B 95 (2017), 144204.

[8] M. Piccardo, C.-K. Li, Y.-R. Wu, J.S. Speck, B. Bonef, R.M. Farrell, M. Filoche, L. Martinelli, J. Peretti, C. Weisbuch, Localization landscape theory of disorder in semiconductors. II. Urbach tails of disordered quantum well layers, Phys. Rev. B 95 (2017), 144205.

[9] C.-K. Li, M. Piccardo, L.-S. Lu, S. Mayboroda, L. Martinelli, J. Peretti, J.S. Speck, C. Weisbuch, M. Filoche, Y.-R. Wu, Localization landscape theory of disorder in semiconductors. III. Application to Carrier transport and recombination in light emitting diodes, Phys. Rev. B 95 (2017), 144206.

[10] C.-L. Tsai, Effects of underlying InGaN/GaN superlattice structures on the structural and optical properties of InGaN LEDs, J. Lumin. 174 (2016) $36-41$.

[11] S.F. Chichibu, A. Uedono, T. Onuma, B.A. Haskell, A. Chakraborty, T. Koyama, P.T. Fini, S. Keller, S.P. Denbaars, J.S. Speck, U.K. Mishra, S. Nakamura, S. Yamaguchi, S. Kamiyama, H. Amano, I. Akasaki, J.H. Han, T. Sota, Origin of defect-insensitive emission probability in In-containing (Al,In,Ga)N alloy semiconductors, Nat. Mater. 5 (2006) 810-816.

[12] H.-S. Chen, C.-F. Lu, D.-M. Yeh, C.-F. Huang, J.-J. Huang, C.-C. Yang, Orange-red light-emitting diodes based on a prestrained InGaN-GaN quantum-well epitaxy structure, IEEE Photon. Technol. Lett. 18 (2006) 2269-2271.

[13] K.P. O'Donnell, R.W. Martin, P.G. Middleton, Origin of luminescence from InGaN diodes, Phys. Rev. Lett. 82 (1999) $237-240$.

[14] P. Dawson, S. Schulz, R.A. Oliver, M.J. Kappers, C.J. Humphreys, The nature of carrier localisation in polar and nonpolar InGaN/GaN quantum wells, J. Appl. Phys. 119 (2016), 181505.

[15] P. Lefebvre, T. Taliercio, S. Kalliakos, A. Morel, X.B. Zhang, M. Gallart, T. Bretagnon, B. Gil, N. Grandjean, B. Damilano, J. Massies, Carrier dynamics in group-III nitride low-dimensional systems: localization versus quantum-confined Stark effect, Phys. Stat. Sol.(b) 228 (2001) 65-72.

[16] M.J. Galtrey, R.A. Oliver, M.J. Kappers, C.J. Humphreys, D.J. Stokes, P.H. Clifton, A. Cerezo, Three-dimensional atom probe studies of an In ${ }_{x} G_{1}{ }_{1-x} N / G a N$ multiple quantum well structure: assessment of possible indium clustering, Appl. Phys. Lett. 90 (2007), 061903.

[17] M.J. Galtrey, R.A. Oliver, M.J. Kappers, C.J. Humphreys, P.H. Clifton, D. Larson, D.W. Saxey, A. Cerezo, Three-dimensional atom probe analysis of greenand blue-emitting $\operatorname{In}_{\mathrm{x}} \mathrm{Ga}_{1-\mathrm{x}} \mathrm{N} / \mathrm{GaN}$ multiple quantum well structures, J. Appl. Phys. 104 (2008), 013524. 
[18] C.J. Humphreys, J.T. Griffiths, F. Tang, F. Oehler, S.D. Findlay, C. Zheng, J. Etheridge, T.L. Martin, P.A.J. Bagot, M.P. Moody, D. Sutherland, P. Dawson, S. Schulz, S. Zhang, W.Y. Fu, T. Zhu, M.J. Kappers, R.A. Oliver, The atomic structure of polar and non-polar InGaN quantum wells and the green gap problem, Ultramicroscopy 176 (2017) 93-98.

[19] A. Hangleiter, F. Hitzel, C. Netzel, D. Fuhrmann, U. Rossow, G. Ade, P. Hinze, Suppression of nonradiative recombination by V-shaped pits in GaInN/GaN quantum wells produces a large increase in the light emission efficiency, Phys. Rev. Lett. 95 (2005), 127402.

[20] A. Hangleiter, C. Netzely, D. Fuhrmanny, F. Hitzely, l. Hoffmanny, H. Bremersy, U. Rossowy, G. Adez, P. Hinzez, Anti-localization suppresses nonradiative recombination in GaInN/GaN quantum wells, Philos. Mag. A 87 (2007), 2041.

[21] J.-H. Ryou, P.D. Yoder, J. Liu, Z. Lochner, H. Kim, S. Choi, H.J. Kim, R.D. Dupuis, Control of quantum-confined Stark effect in InGaN-based quantum wells, IEEE J. Sel. Top. Quantum Electron. 15 (2009) 1080-1091.

[22] T. Takeuchi, S. Sota, M. Katsuragawa, M. Komori, H. Takeuchi, H. Amano, I. Akasaki, Quantum-confined Stark effect due to piezoelectric fields in GaInN strained quantum wells, Jpn. J. Appl. Phys. 36 (1997) L382-L385.

[23] N. Nanhui, W. Huaibing, L. Jianping, L. Naixin, X. Yanhui, H. Jun, D. Jun, S. Guangdi, Enhanced luminescence of InGaN/GaN multiple quantum wells by strain reduction, Solid State Electron. 51 (2007) 860-864.

[24] M.B. Nardelli, K. Rapcewicz, J. Bernholc, Polarization field effects on the electron-hole recombination dynamics in $\mathrm{In}_{0.2} \mathrm{Ga}_{0.8} \mathrm{~N} / \mathrm{In}_{1-\mathrm{x}} \mathrm{Ga}_{\mathrm{x}} \mathrm{N}$ multiple quantum wells, Appl. Phys. Lett. 71 (1997) 3135-3137.

[25] S. Alam, S. Sundaram, M. Elouneg-Jamroz, X. Li, Y. El Gmili, I.C. Robin, P.L. Voss, J.-P. Salvestrini, A. Ougazzaden, InGaN/InGaN multiple-quantum-well grown on InGaN/GaN semi-bulk buffer for blue to cyan emission with improved optical emission and efficiency droop, Superlattices Microstruct. 104 (2017) 291-297.

[26] L. Zhang, K. Cheng, H. Liang, R. Lieten, M. Leys, G. Borghs, Photoluminescence studies of polarization effects in InGaN/(In)GaN multiple quantum well structures, Jap. J. Appl. Phys. 51 (2012), 030207.

[27] C.-F. Huang, C.-Y. Chen, C.-F. Lu, C.C. Yang, Reduced injection current induced blueshift in an InGaN/GaN quantum-well light-emitting diode of prestrained growth, Appl. Phys. Lett. 91 (2007), 051121.

[28] C. Lu, L. Wang, J. Lu, R. Li, L. Liu, D. Li, N. Liu, L. Li, W. Cao, W. Yang, W. Chen, W. Du, C.-T. Lee, X. Hu, Investigation of the electroluminescence spectrum shift of InGaN/GaN multiple quantum well light-emitting diodes under direct and pulsed currents, J. Appl. Phys. 113 (2013), 013102.

[29] L. Liu, L. Wang, D. Li, N. Liu, L. Li, W. Cao, W. Yang, C. Wan, W. Chen, W. Du, X. Hu, Z.C. Feng, Influence of indium composition in the prestrained InGaN interlayer on the strain relaxation of InGaN/GaN multiple quantum wells in laser diode structures, J. Appl. Phys. 109 (2011), 073106.

[30] Y.-S. Chen, L.-C. Yao, Y.-L. Lin, L. Hung, C.-F. Huang, T.-Y. Tang, J.-J. Huang, W.Y. Shiao, C.C. Yang, Transmission electron microscopy study on pre-strained InGaN/GaN quantum wells, J. Cryst. Growth 297 (2006) 66-73.

[31] C.-F. Huang, T.-C. Liu, Y.-C. Lu, W.-Y. Shiao, Y.-S. Chen, J.-K. Wang, C.-F. Lu, C.C. Yang, Enhanced efficiency and reduced spectral shift of green lightemitting-diode epitaxial structure with prestrained growth, J. Appl. Phys, 104 (2008), 123106.

[32] C.-F. Huang, T.-Y. Tang, J.-J. Huang, W.-Y. Shiao, C.C. Yang, C.-W. Hsu, L.C. Chen, Prestrained effect on the emission properties of InGaN/GaN quantumwell structures, Appl. Phys. Lett. 89 (2006), 051913.

[33] W.-Y. Shiao, C.-F. Huang, T.-Y. Tang, J.-J. Huang, Y.-C. Lu, C.-Y. Chen, Y.-S. Chen, C.C. Yang, X-ray diffraction study on an InGaN/GaN quantum-well structure of prestrained growth, J. Appl. Phys. 101 (2007), 113503.

[34] Y. Takahashi, A. Satake, K. Fujiwara, J.K. Shue, U. Jahn, H. Kostial, H.T. Grahn, Enhanced radiative efficiency in blue (In,Ga)N multiple-quantum-well light-emitting diodes with an electron reservoir layer, Physica E 21 (2004) 876-880.

[35] S.J. Leem, Y.C. Shin, K.C. Kim, E.H. Kim, Y.M. Sung, Y. Moon, S.M. Hwang, T.G. Kim, The effect of the low-mole InGaN structure and InGaN/GaN strained layer superlattices on optical performance of multiple quantum well active layers, J. Cryst. Growth 311 (2008) 103-106.

[36] T. Li, Q.Y. Wei, A.M. Fischer, J.Y. Huang, Y.U. Huang, F.A. Ponce, J.P. Liu, Z. Lochner, J.-H. Ryou, R.D. Dupuis, The effect of InGaN underlayers on the electronic and optical properties of InGaN/GaN quantum wells, Appl. Phys. Lett. 102 (2013), 041115.

[37] N. Nanhui, W. Huaibing, L. Jianping, L. Naixin, X. Yanhui, H. Jun, D. Jun, S. Guangdi, Improved quality of InGaN/GaN multiple quantum wells by a strain relief layer, J. Cryst. Growth 286 (2006) 209-212.

[38] J.K. Sheu, G.C. Chi, M.J. Jou, Enhanced output power in an InGaN-GaN multiquantum-well light-emitting diode with an InGaN current-spreading layer, IEEE Photon. Technol. Lett. 13 (2001) 1164-1166.

[39] Z. Zheng, Z. Chen, Y. Xian, B. Fan, S. Huang, W. Jia, Z. Wu, G. Wang, H. Jiang, Role of InGaN insertion layer on nitride-based light-emitting diodes, Jap. J. Appl. Phys. 51 (2012), 072101.

[40] Ü. Özgür, X. Ni, X. Li, J. Lee, S. Liu, S. Okur, V. Avrutin, A. Matulionis, H. Morkoç, Ballistic transport in InGaN-based LEDs: impact on efficiency, Semicond. Sci. Technol. 26 (2011), 014022.

[41] V. Avrutin, S.A. Hafiz, F. Zhang, Ü. Özgür, H. Morkoç, A. Matulionis, InGaN light-emitting diodes: efficiency-limiting processes at high injection, J. Vac. Sci. Technol. A 31 (2013), 050809.

[42] X. Ni, X. Li, J. Lee, S. Liu, V. Avrutin, Ü. Özgür, H. Morkoç, A. Matulionis, T. Paskova, G. Mulholland, K.R. Evans, InGaN staircase electron injector for reduction of electron overflow in InGaN light emitting diodes, Appl. Phys. Lett. 97 (2010), 031110.

[43] V. Avrutin, S.A. Hafiz, F. Zhang, Ü. Özgür, E. Bellotti, F. Bertazzi, M. Goano, A. Matulionis, A.T. Roberts, H.O. Everitt, H. Morkoç, Saga of efficiency degradation at high injection in InGaN light emitting diodes, Turk. J. Phys. 38 (2014) 269-313.

[44] G. Schmidt, M. Muller, P. Veit, F. Bertram, J. Christen, M. Glauser, J.-F. Carlin, G. Cosendey, R. Butte, N. Grandjean, Nano-scale luminescence characterization of individual InGaN/GaN quantum wells stacked in a microcavity using scanning transmission electron microscope cathodoluminescence, Appl. Phys. Lett. 105 (2014), 032101.

[45] J.-H. Ryou, W. Lee, J. Limb, D. Yoo, J.P. Liu, R.D. Dupuis, Z.H. Wu, A.M. Fischer, F.A. Ponce, Control of quantum-confined Stark effect in InGaN/GaN multiple quantum well active region by p-type layer for III-nitride-based visible light emitting diodes, Appl. Phys. Lett. 92 (2008), 101113.

[46] C. Zhao, R. Zhang, B. Liu, D. Fu, M. Li, X. Xiu, Z. Xie, Y. Zheng, A modified simplified coherent potential approximation model of band gap energy of III-V ternary alloys, Sci. China Phys. Mech. Astron. 55 (2012) 400-403.

[47] Y.-H. Ra, R. Navamathavan, J.-H. Park, C.-R. Lee, Radial growth behavior and characteristics of m-plane In0.16Ga0.84N/GaN MQW nanowires by MOCVD, Cryst. Eng. Comm. 15 (2013), 1874-188.

[48] Y.-T. Moon, H.H. Lee, D.Y. Noh, S.-J. Park, D.-J. Kim, J.-S. Park, J.-T. Oh, Growth-temperature dependent property of GaN barrier layer and its effect on InGaN/GaN multiple quantum well light-emitting diodes, J. Korean Phys. Soc. 42 (2003) 557-561.

[49] D. Holec, P.M.F.J. Costa, M.J. Kappers, C.J. Humphreys, Critical thickness calculations for InGaN/GaN, J. Cryst. Growth 303 (2007) $314-317$.

[50] D. Holec, Y. Zhang, D.V. Sridhara Rao, M.J. Kappers, C. McAleese, C.J. Humphreys, Equilibrium critical thickness for misfit dislocations in III-nitrides, J. Appl. Phys. 104 (2008), 123514. 